\title{
Contribution to the knowledge of tomentelloid fungi in the Ilberian Peninsula. II.
}

\author{
IRENEIA MELO, ISABEL SALCEDO AND MARLA TERESA TELLERÍA
}

\begin{abstract}
MELO, I., SALCEDO, I. \& TELLERÍA, M.T. 2000: Contribution to the knowledge of tomentelloid fungi in the Iberian Peninsula. II. - Karstenia 40: 93-101. Helsinki. ISSN 0453-3402.

Descriptions and original iconography are given for the following tomentelloid basidiomycetes from the Iberian Peninsula: Tomentella badia, T. cinereoumbrina, T. fuscocinerea, T. bresadolae, T. bryophila, T. brevispina, T. neobourdotii, T. ramosissima and $T$. stuposa. Their distribution in the area is reviewed. T. brevispina is a new record to the Iberian Peninsula. T. badia, T. fuscocinerea, T. bryophila and T. neobourdotii are new to Portugal.
\end{abstract}

Key words: chorology, Iberian Peninsula, Tomentella

Ireneia Melo, Museu e Jardim Botanico da Universidade de Lisboa, Rua da Escola Politécnica 58, P-1250-102 Lisboa, Portugal

Isabel Salcedo, Depto. de Biología Vegetal y Ecología (Botánica), Universidad del País Vasco, Aptdo. 644, E- 48080 Bilbao, Spain

Maria Teresa Telleria, Real Jardín Botánico, CSIC, Plaza de Murillo 2, E-28014 Madrid, Spain

\section{Introduction}

This is a continuation of a study of tomentelloid fungi from the Iberian Peninsula started recently (Melo et al. 1998). The first contribution dealt mainly with cystidioid and/ or dimitic species. The present issue comprises afibulate species as well as species with globose to subglobose spores.

For the simple-septate species we have followed the opinion of Kõljalg (1996) and for the fibulate ones Larsen (1974), because the Iberian material of this group fits better with the species concept of the latter author.

The problem of the species delimitation in the genus Tomentella could be resolved with the help of complementary modern molecular techniques we know are being developed (Kõljalg 1999). Nevertheless, we think that studies involving morphological and chorological data are still necessary to improve the general knowledge of this controversial genus.

\begin{abstract}
Material and methods
The descriptions of species were based on the specimens deposited in BIO, LISU and MA. Citation of the authors of the scientific names follows Brummitt \& Powell (1992). Measurements and drawings were made from microscopical sections mounted in $3 \% \mathrm{KOH}$. A Leitz Dialux $20 \mathrm{~EB}$ microscope provided with a drawing tube was used to make the drawings. The colour terms are from the Munsell Soil Color Charts (1990). In the specimen data the following abbreviations are used: IM= Ireneia Melo, IS= Isabel Salcedo, MD = Margarita Dueñas, MT = Maria Teresa Tellería.
\end{abstract}

\section{Descriptions of species}

Tomentella badia (Link) Stalpers, Rev. Mycol. 29: 98 . 1975. - Fig. 1

三Sporotrichum badium Link, Mag. Ges. Naturf. Freunde 3: 12. 1809.

Basidioma resupinate, adherent to the substrate, continuous, more or less mucedinoid to arachnoid. Hymenophore smooth, dark grayish brown 


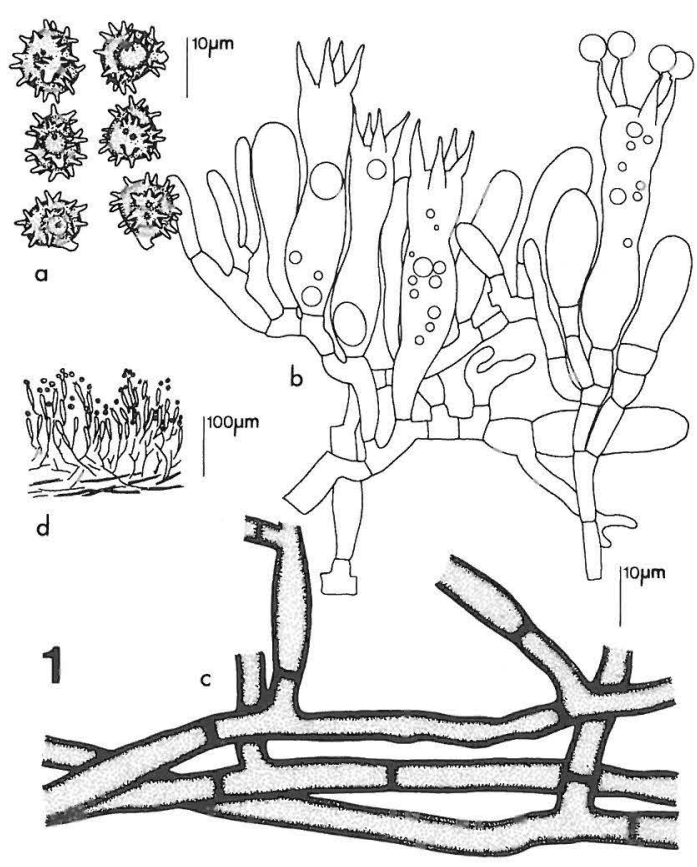

Fig. 1. Tomentella badia (I Melo 6742, LISU): a. basidiospores, b. basidia and subhymenial hyphae, c. subicular hyphae, d. section through basidioma.
(10YR 4/2) to dark brown (7.5YR 4/3). Subiculum arachnoid, darker than the hymenophore; cordons absent. Margin indeterminate. Hyphal system monomitic; subicular hyphae without clamps, 4-8 $\mu \mathrm{m}$ diam., thick-walled, brown; subhymenial hyphae without clamps, 3-4 $\mu \mathrm{m}$ diam., thin to slightly thick-walled, hyaline to sometimes pale brown. Subhymenial and hymenial hyphae often with bluish green diffusates in $3 \% \mathrm{KOH}$. Basidia clavate, sinuous, (35-)40-75 $\times$ (7-) $8-10 \mu \mathrm{m}$, with 4 up to $8 \mu \mathrm{m}$ long sterigmata. Basidiospores subglobose to irregularly globose, $8-11 \times 7-8(-10) \mu \mathrm{m}$, aculeate, spines up to $2 \mu \mathrm{m}$ long, pale brown.

Remarks: - This species can be distinguished by simple septate hyphae and irregularly globose spores with long spines. Previously reported only from Spain, Huelva (Tellería 1980, as $T$. atroviolacea Litsch.), Menorca (Tellería et al. 1997) and Valencia (Malençon \& Bertault 1971, as Tomentella spongiosa (Schwein. : Fr.) Höhn. \& Litsch.). New to Portugal.

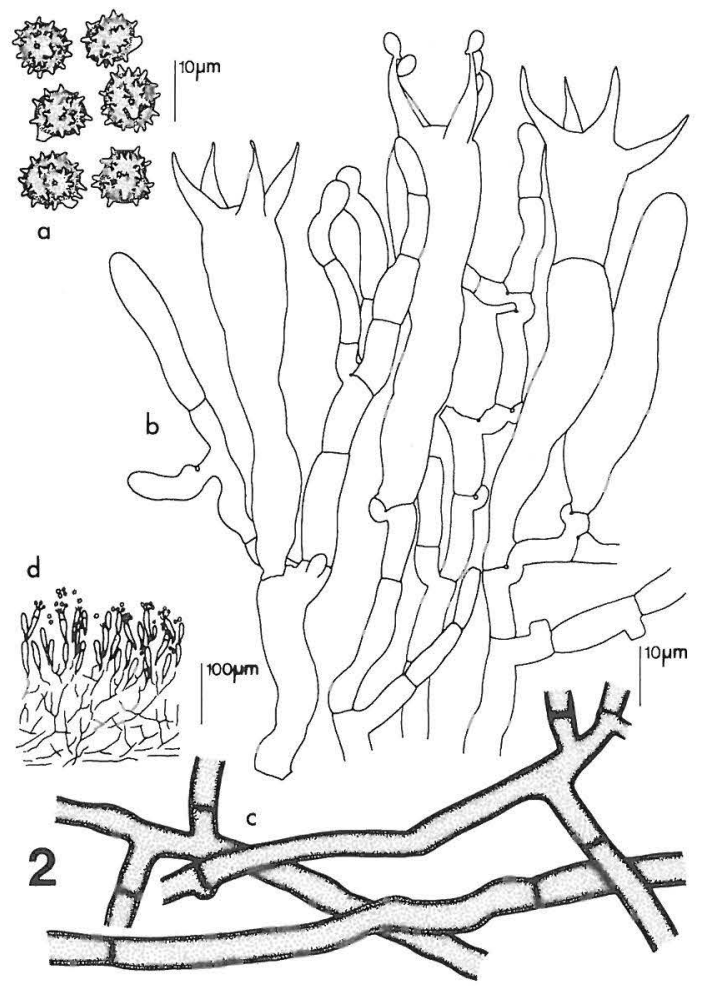

Fig. 2. Tomentella cinereoumbrina (I. Melo 5213, LISU): a. basidiospores, b. basidia and subhymenial hyphae, c. subicular hyphae, d. section through basidioma.

Material studied: Portugal. Ribatejo, Ferreira do Zêzere, Pereiro, 29SND5499, on Crataegus monogyna Jacq., 11.IX.1997 IM 7196 (LISU). Spain. Alava, San Millán, Aspuru, 30TWN4552, $600 \mathrm{~m}$, on Quercus pyrenaica Willd., 29.VII.1987 IS 3974 (BIO-Fungi 891). Cantabria, Los Tojos, de Bárcena Mayor al cruce de los Tojos, junto al río Argoza Labor, 30TVN0179, 570 m, 9.X.1986 MT 7782 (MA-Fungi 18986). Gerona, Pals, Pinedes dels Masos de Pals, 31TEG1548, $40 \mathrm{~m}$, on Pinus sp., 9.XI.1995 IM 6742 (LISU). Jaén, Cazorla, Vara del Espino, 30SWG0895, $1450 \mathrm{~m}$, on Pinus nigra J.F. Arnold, 11.V.1990 IM 4838 (LISU).

Tomentella cinereoumbrina (Bres.) Stalpers, Stud. Mycol. 35: 26. 1993. - Fig. 2

$\equiv$ Hypochnus cinereoumbrinus Bres., Stud. Trent. ser. II, 7: 62. 1926.

Basidioma resupinate, adherent to the substrate, continuous, crustose. Hymenophore smooth, brown (7.5YR 5/3) to dark brown (10YR 4/3). Subiculum dense, concolorous to slightly darker than the hymenophore; without cordons. Margin indeterminate. 


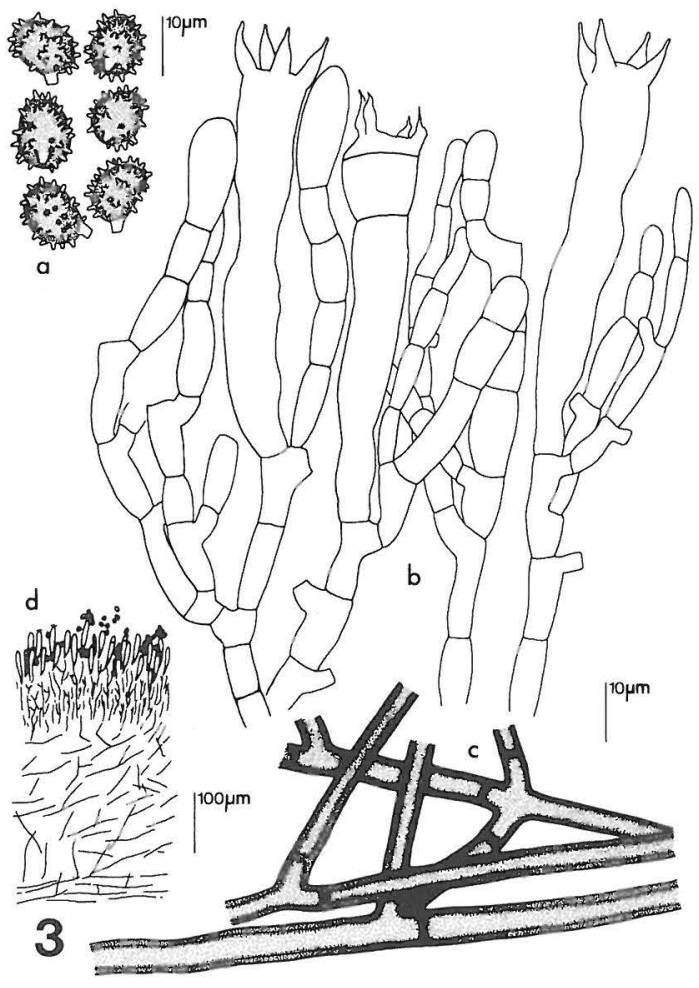

Fig. 3. Tomentella fuscocinerea (I. Melo 7195, LISU): a. basidiospores, b. basidia and subhymenial hyphae, c. subicular hyphae, d. section through basidioma.

Hyphal system monomitic; subicular hyphae without clamps, sometimes showing scattered clamps, 3-6 $\mu \mathrm{m}$ diam., slightly thick-walled, yellowish brown; subhymenial hyphae without clamps, sometimes with few clamps, 3-5 $\mu \mathrm{m}$ diam., thin-walled, hyaline. Subhymenial and hymenial hyphae often with bluish green diffusates in $3 \% \mathrm{KOH}$. Basidia clavate to utriform, often with transverse septa, 50-75 $\times 9-12 \mu \mathrm{m}$, with 4 up to $12 \mu \mathrm{m}$ long sterigmata. Basidiospores subglobose, (8-)9-12 × 7-9 $\mu \mathrm{m}$, echinulate, pale brown to brown.

Remarks: The presence of scattered clamps on the subicular and subhymenial hyphae and the subglobose and short-spined (up to $1 \mu \mathrm{m}$. spores enable the identification of this species. So far only reported from two areas in the Iberian Peninsula: Portugal, Estremadura (Torrend 1913, as Hypochnus crustaceus (Schumach. : Fr.) P.Karst. sensu Bres.) and Spain (Larsen 1981, without indication of locality).
Material studied: Spain. Avila, Piedralaves, 30TUK5665, $630 \mathrm{~m}$, on burnt wood of Pinus pinea L., 12.XII.1983 MT 4789bis (MA-Fungi 7351). Menorca, Ciutadella, La Vall, 31TEE8833, $20 \mathrm{~m}$, on Pinus halepensis Mill., 17.XI.1990 IM 5213, 5214 (LISU).

Tomentella fuscocinerea (Pers.) Donk, Med. Bot. Mus. Herb. Rijks-Univ. Utrecht 9: 30. 1933.Fig. 3

$\equiv$ Thelephora fuscocinerea Pers., Mycol. Europ. 1: 114. 1822.

Basidioma resupinate, adherent, continuous, crustose, cracked when dried. Hymenophore smooth, dark brown (7.5YR 4/2). Subiculum dense, fibrous, concolorous; without cordons. Margin byssoid, white (10YR $8 / 2)$ to very pale brown (10YR 7/3).

Hyphal system monomitic; subicular hyphae without clamps, 4-6 $\mu \mathrm{m}$ diam., thick-walled, brown; subhymenial hyphae without clamps, 3-5 $\mu \mathrm{m}$ diam., often densely ramified with short and inflated cells, thin to slightly thick-walled, hyaline to pale brown. Sometimes subhymenial and hymenial hyphae with bluish green diffusates in $3 \% \mathrm{KOH}$. Basidia clavate to utriform, sinuous, $45-75 \times 8-12 \mu \mathrm{m}$, with 4 up to $8 \mu \mathrm{mlong}$ sterigmata. Basidiospores subglobose to ellipsoid, 9-12 × 7-9 $\mu \mathrm{m}$, echinulate, brown.

Remarks: This species is easily recognized by dense and crustose basidioma with pale margin, hyphae without clamps and subhymenial hyphae with short and inflated cells. New to Portugal. Known so far from Northeast and South Spain: Granada (Tellería 1985, as Tomentellastrum alutaceo-umbrinum (Bres.) Larsen), Lérida and Tarragona (Llimona et al. 1998).

Material studied: Portugal. Ribatejo, Ferreira do Zêzere, Pereiro, 29SND5499, on Crataegus monogyna Jacq.,11.IX.1997 IM 7195 (LISU). Spain. Alava, Cigoitia, Apodaca, 30TWN2152, $550 \mathrm{~m}$, on Juniperus sp., 31.X.1987 IS 4114 (BIO-Fungi 3387); idem, IS 4148 bis (BIO-Fungi 926); Maeztu, El Ayedo, 30TWN4629, $850 \mathrm{~m}$, on Thymus sp., 6.XII.1987 IS 4232 bis (BIOFungi 843); ibidem, on Quercus rotundifolia Lam., 6.XII.1987 IS 4263 (BIO-Fungi 2101); Laguardia, entre Laguardia y Oyón, 30TWN3709, 500 m, on Rosmarinus officinalis L., 7.XII.1987 IS 4380 (BIO-Fungi 2125). Cuenca, Sierra Altomira, Illana, 30TWK1148, on Rosmarinus officinalis L., 28.IV.1988 MD 4375 (MA-Fungi 21326). Granada, Alhama de Granada, 30SVF0991, on Quercus rotundifolia Lam., 19.I.1982 (MA-Fungi 3805). Guadalajara, Monte de la Alcarria, de Horche a Yebes, 30TVK9188, on Thymus sp., 1.XI.1987 MD 3827 (MA-Fungi 21287-2); Sigüenza, 


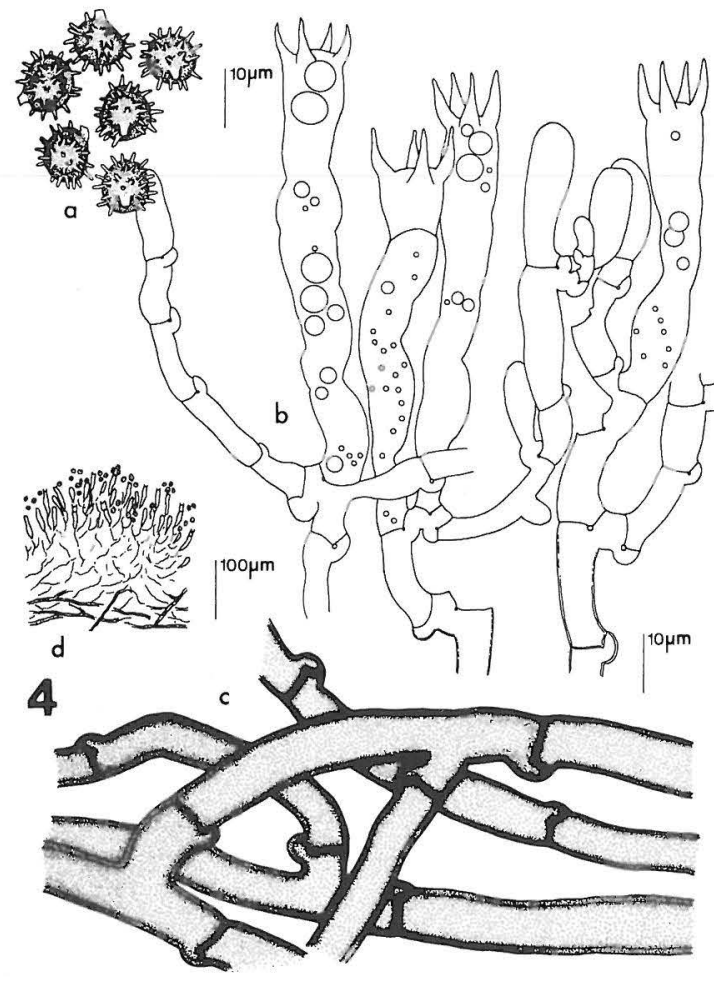

Fig. 4. Tomentella bresadolae (I. Melo 6655, LISU): a. basidiospores, b. basidia and subhymenial hyphae, c. subicular hyphae, d. section through basidioma.

30TWL3047, on Thymus sp., 5.X.1987 (MA-Fungi 24519). Menorca, Mahón, S' Albufera, 31SFE0823, on Quercus ilex L., 15.XI.1990 IM 5108 (LISU); Mercadal, Binifamis, 31SFE0128, $60 \mathrm{~m}$, 15.XI.1990 IM 5075 (LISU). Vizcaya, Txatxarramendi, 30TWP2505, on mosses, 9.III.1986 IS 1913 (BIO-Fungi 7947).

Tomentella bresadolae (Brinkmann) Bourdot \& Galzin, Bull. Soc. Mycol. France 40: 155. 1924.Fig. 4

झ Hypochnus bresadolae Brinkmann in Bres., Ann. Mycol. 1: 108. 1903.

Basidioma resupinate, adherent, continuous to discontinuous, more or less mucedinoid to arachnoid. Hymenophore smooth to minutely granulose, brown $(7.5 \mathrm{YR} \mathrm{5/2)}$ to dark brown (7.5YR 4/2). Subiculum arachnoid, darker than hymenophore; without cordons. Margin indeterminate. Hyphal system monomitic; subicular hyphae with clamps, 8-9 $\mu \mathrm{m}$ diam., thick-walled, dark brown; subhymenial hyphae with clamps, 4-6 $\mu \mathrm{m}$ diam., thin to slightly thick-walled, hyaline to pale brown. Subhymenial and hymenial hyphae often with bluish green diffusates in $3 \% \mathrm{KOH}$. Basidia clavate, sinuous, sometimes with transverse septa, $60-70(-75) \times 10-11 \mu \mathrm{m}$, with 4 up to $8 \mu \mathrm{m}$ long sterigmata. Basidiospores globose, $8-9 \mu \mathrm{m}$ diam., aculeate, brown.

Remarks: Microscopically this species is very similar to T. brevispina, T. bryophila and T. stuposa. T. brevispina has subhymenial hyphae with short and inflated cells and narrower subicular hyphae. The yellowish brown globose spores make $T$. bryophila easily recognized. The characteristic more evident in $T$. stuposa is the strong swelling of the cell walls in $\mathrm{KOH}$. In the Iberian literature this species has been confounded with $T$. brevispina and T. bryophila. So, in spite of being previously widely reported from this area, we were only able to confirm the collections studied.

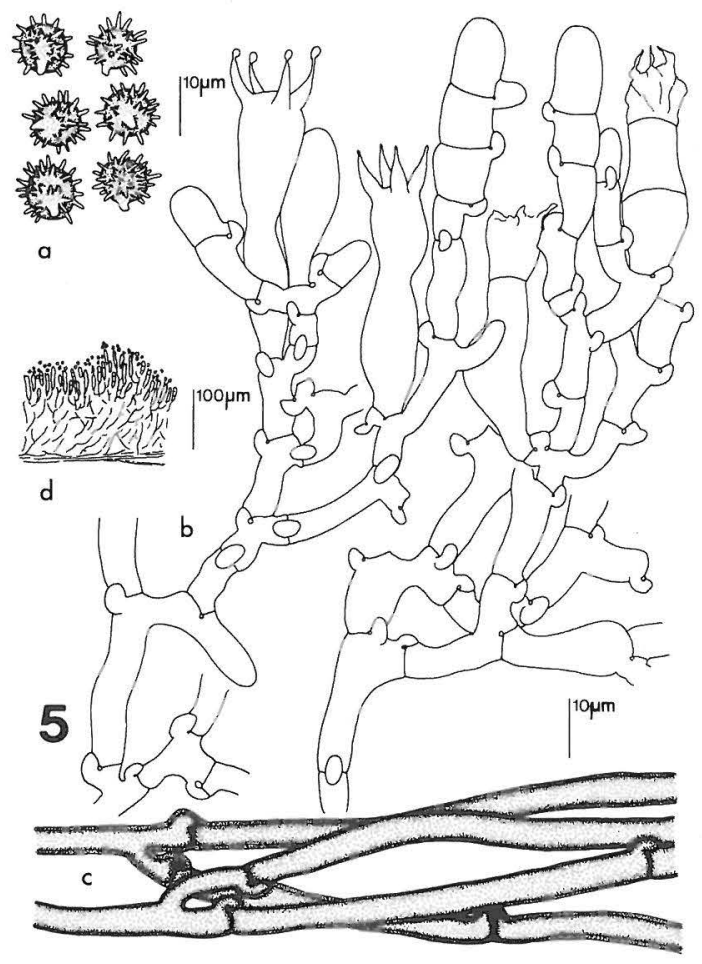

Fig. 5. Tomentella brevispina (I. Melo 7018, LISU): a. basidiospores, b. basidia and subhymenial hyphae, c. subicular hyphae, d. section through basidioma. 
Material studied: Portugal. Algarve, Monchique, entre Caldas de Monchique e Nave, 29SNB3827, 290 m, on Quercus suber L., 24.I.1990 IM 4632 (LISU). Spain. Gerona, Parque Natural de l'Albera, junto al castillo de Requesens, 31TDG9599, $460 \mathrm{~m}$, on Quercus ilex L.), 7.XI.1995 IM 6655 (LISU).

Tomentella brevispina (Bourdot \& Galzin) M.J. Larsen, Mycologia 62: 136. 1970. - Fig. 5

$\equiv$ Tomentella spongiosa var. brevispina Bourdot \& Galzin, Bull. Soc. Mycol. France 40: 154. 1924.

Basidioma resupinate, adnate to the substrate, continuous, mucedinoid. Hymenophore smooth or slightly granulose, reddish brown (5YR 4/3, 4/ 4) to dark reddish brown (5YR 3/3, 3/4). Subiculum arachnoid to fibrous, concolorous; sometimes with narrow cordons. Margin indeterminate. Hyphal system monomitic; subicular hyphae sometimes forming narrow cordons, with clamps, 3-6 $\mu \mathrm{m}$ diam., thick-walled, brown; subhymenial hyphae with clamps, 3-5 $\mu \mathrm{m}$ diam., thin-walled, densely ramified with short and inflated cells, up to $8 \mu \mathrm{m}$ diam., pale brown. Subhymenial and hymenial hyphae sometimes with green reaction in $3 \% \mathrm{KOH}$. Basidia cylindrical to subclavate, sinuous, 35-50 $\times 10-11 \mu \mathrm{m}$, with 4 up to $7 \mu \mathrm{m}$ long sterigmata. Basidiospores globose, 7-8 (-9) $\mu \mathrm{m}$, echinulate to aculeate, pale brown.

Remarks: Similar to T. bresadolae, however the narrower subicular hyphae and the densely ramified subhymenial ones, with short and inflated cells, distinguish T. brevispina. New record to the Iberian Peninsula.

Material studied: Portugal. Algarve, Monchique, entre Caldas de Monchique e Nave, 29SNB3827, 290 m, on Quercus suber L., 24.I.1990 IM 4631 (LISU). Beira Alta, Oliveira de Frades, Arca, Gandra, 29TNE6795, on Quercus robur L., 5.XI.1996 IM 7017-7019, 7027 (LISU). Spain. Asturias, Reserva Biológica de Muniellos, Tablizas, 29TPH8868, on Quercus petraea (Matt.) Liebl., 18.X.1983 MD 1184 (MA-Fungi 14629); idem, MD 1236 (MA-Fungi 14631). Cádiz, pinsapar de Benamahona, 30STF8173, on unidentified wood, (MAFungi 825). Huesca, Parque Nacional de Ordesa, Cotatuero, junto a la Virgen de Ordesa, 30TYN4125, $1350 \mathrm{~m}$, on coniferous wood, 16.X.1989 IS 7396 (BIO-Fungi 5633); ibidem, on Abies alba Mill., 16.X.1989 MD 5221 (MA-Fungi 26357). Vizcaya, bajada de Urkiola a Ochandiano, 30TWN2870, on Larix sp., 9.X.1983 IS 414 (MA-Fungi 7155); Kolitxa, 30VN7983, $630 \mathrm{~m}$, on Quercus robur L., 10.X.1983 IS 494 (BIO-Fungi 3152).

Tomentella bryophila (Pers.) M.J. Larsen, Mycol. Mem. 4: 51. 1974. - Fig. 6

$\equiv$ Sporotrichum bryophilum Pers., Mycol. Europ. 1: 78. 1822.

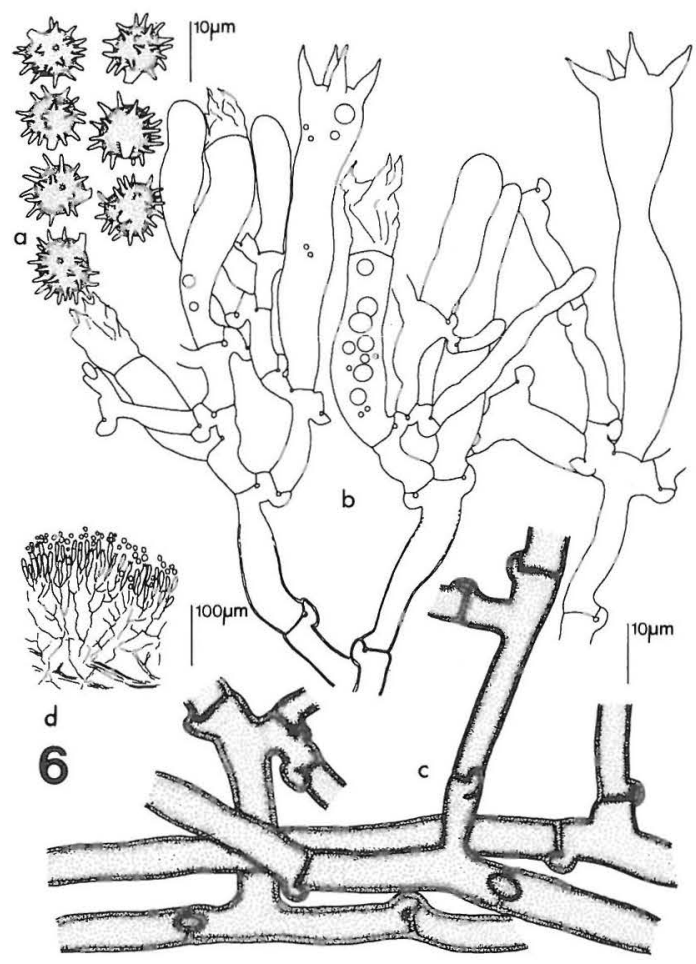

Fig. 6. Tomentella bryophila (I. Melo 6253, LISU): a. basidiospores, b. basidia and subhymenial hyphae, c. subicular hyphae, d. section through basidioma.

Basidioma resupinate, adherent, continuous, more or less mucedinoid. Hymenophore smooth, strong brown $(7.5 Y R 4 / 6,5 / 6)$ to yellowish brown (10YR5/8). Subiculum arachnoid to fibrous, darker than the hymenophore; without cordons. Margin indeterminate. Hyphal system monomitic; subicular hyphae with clamps, 4-7 $\mu \mathrm{m}$ diam., thick-walled, dark brown; subhymenial hyphae with clamps, 4-6 $\mu \mathrm{m}$ diam., thin to slightly thickwalled, pale yellowish brown. Basidia cylindrical to clavate, sinuous, $55-65 \times 9-12 \mu \mathrm{m}, 4$ up to 10 $\mu \mathrm{m}$ long sterigmata. Basidiospores globose, 8 $10 \mu \mathrm{m}$ diam., aculeate, spines up to $2.5 \mu \mathrm{m}$ long., bright yellowish brown.

Remarks: The ferruginous basidioma and the yellowish brown aculeate globose spores, are characteristics that make this species easily recognized. Widespread in Spain: Asturias (Dueñas \& Tellería 1988), Barcelona (Bertault 1982, Tellería 
1984, Llimona et al. 1998), Ciudad Real (Dueñas 1991), Cáceres (Blanco Bueno 1991), Huesca (Hjortstam et al. 1981; Melo et al. 1993), León ( Dueñas \& Tellería 1988), Mallorca (Tellería 1985), Navarra (Hjortstam et al. 1981), Soria (Tellería 1980; Hjortstam et al. 1981), Tarragona (Llimona et al. 1998) and Toledo (Dueñas 1991). New to Portugal.

Material studied: Portugal. Algarve, S. Bras de Alportel, E.N. 2, km 713, 29SNB9418, on Quercus suber L., 23.I.1990 IM 4564, 4566 (LISU). Baixo Alentejo, Barrancos, Santo Aleixo da Restauraçao, 29SPC6115, $235 \mathrm{~m}$, on unidentified wood, 12.XII.1994 IM 6457 (LISU). Spain. Alava, Cigoitia, Apodaca, 30TWN2152, $550 \mathrm{~m}$, on Juniperus communis L., 31.X.1987 IS 4113 (BIO-Fungi 934); idem, IS 4148 (BIO-Fungi 909); ibidem, on Quercus rotundifolia Lam., 31.X.1987 IS 4134 (BIO-Fungi 918); Laguardia, entre Laguardia y Oyón, 30TWN3709, 500 m, on Vitis vinifera L., 7.XII.1987 IS 4401 (BIO-Fungi 856). Asturias, Peñamellera Alta, Llonín, 30TUN6699, 8.X.1986 MT 7441 (MA-Fungi 19014); Reserva Biológica de Muniellos, Tablizas, 29TPH8868, on Quercus petraea (Matt.) Liebl., 18.X.1983 MD 1171 (MA-Fungi 14638); ídem, MT 3641 (MA-Fungi 14640). Barcelona, Cabrils, 31TDF4797, on Quercus suber L., 31.X.1979 MT 162. 79 (MA-Fungi 5687). Cantabria, Hermandad de Campoo de Suso, La Lomba, 30TUN9364, on Fagus sylvatica L., 17.XI.1982 MD 443 (MA-Fungi 14635); Los Tojos, de Bácena Mayor al cruce de los Tojos, junto al río Argoza Labor, 30TVN0179, 570 m, MT 7768 (MAFungi 18970); Los Tojos, Saja, hacia el Puerto de Palombera, 30TUN9547, 9.X.1986 MT 7821 (MA-Fungi 18930). Ciudad Real, Sierra del Chorito, de Retuerta de Bullaque a Horcajo de los Montes, 30SUJ7666, on Quercus suber L., 12.V.1988 MD 4671 (MA-Fungi 22463); idem, MD 4677 (MA-Fungi 22469). Granada, Alhama de Granada, 30SVF0991, on Quercus rotundifolia Lam., 12.XII.1980 (MA-Fungi 3791). Huesca, Selva de Oza, 30TXN8941, 1160 m, on Abies alba Mill., 10.XI.1977 MT 757/77 (MA-Fungi 3681); Parque Nacional de Ordesa y Monte Perdido, Bielsa, ermita de Nuestra Señora de Pineta, 31TBH6130, 1300m, on Fagus sylvatica L., 17.X.1989 MD 5277 (MA-Fungi 26404). León, Carretera Nacional VI, entre Villafranca del Bierzo y Pereje, $\mathrm{km} 415,29 \mathrm{TQH} 7822,630 \mathrm{~m}$, on Castanea sativa Mill., 12.XII.1984 MD 3280 (MA-Fungi 14634); idem, $M D$ 3283 (MA-Fungi 14639); MT 6701 (MA-Fungi 14637); idem, MT 6702 (MA-Fungi 14641); Fabero, San Pedro de Paradela, 29TPH9544, on Erica arborea L., 12.XII.1984 MD 3369 (MA-Fungi 14687); Posada de Valdeón, del Collado de Panderruedas a Posada de Valdeón, 30TUN4076, $1220 \mathrm{~m}, 20 . X .1992$ IM 5774 (LISU). Lugo, Becerrea, Vilachá, carretera de Becerrea a Navia de Suarna, km 12, 29TPH5750, 400 m, on Quercus pyrenaica Willd., 4.X.1994 IM 6246 (LISU); ibidem, on Pinus sylvestris L., 4.X.1994 IM 6253 (LISU). Madrid, Miraflores de la Sierra, 30TVL3518, on Quercus pyrenaica Willd., 23.XI.1979 (MA-Fungi 1144). Mallorca, carretera comarcal 710, km 39, 31SDE7905, on Quercus ilex L., 28.III.1984 MT 5265 (MA-Fungi 8504). Navarra, Imoz, Zarranz, 30TWN9755, $700 \mathrm{~m}$, on Quercus sp., 12.X.1990 IS 7603 (BIO-Fungi 216); Lecumberri, a $3 \mathrm{~km}$ en dirección a Ururzun, 30TWN9062, $590 \mathrm{~m}$, on Quercus sp., 11.XI.1977 MT 838/77 (MA-Fungi 3373); prox. de Zabaleta, 30TXN2133, $460 \mathrm{~m}$, on unidentified wood, 8.XI.1977 MT 449/77 (MA-Fungi 3713). Palencia, Cervera de Pisuerga, 30TUN7747, on Quercus pyrenaica Willd., 30.XI.1984 MT 6450 (MA-Fungi 14688); idem, $M T$ 6452 (MA-Fungi 14633); idem, MT 6462 (MA-Fungi 14636). Toledo, Carretera comarcal 508, entre Fresnedilla y el Real de San Vicente, 30TUK5746, on Castanea sativa Mill., 29.V.1988 MD 4733 (MA-Fungi 22510); de Navahermosa a Navas de Estena, Risco de las Paradas, 30SUJ7184, on Quercus pyrenaica Willd., 12.V.1988 MD 4643 (MA-Fungi 23528); prox. de la Iglesuela, 30TUK5256, on Juniperus sp., 21.II.1988 MD 4119 (MA-Fungi 23007). Vizcaya, Somorrostro, Monte Montano, 30TVN9298, $200 \mathrm{~m}$, on Quercus robur L., 3.IX.1983 IS 279 (BIO-Fungi 8412).

Tomentella neobourdotii M.J. Larsen, Mycologia 60: 1179. 1969. - Fig. 7

Basidioma resupinate, adnate to separable from the substrate, continuous, more or less mucedinoid. Hymenophore granulose, dark brown

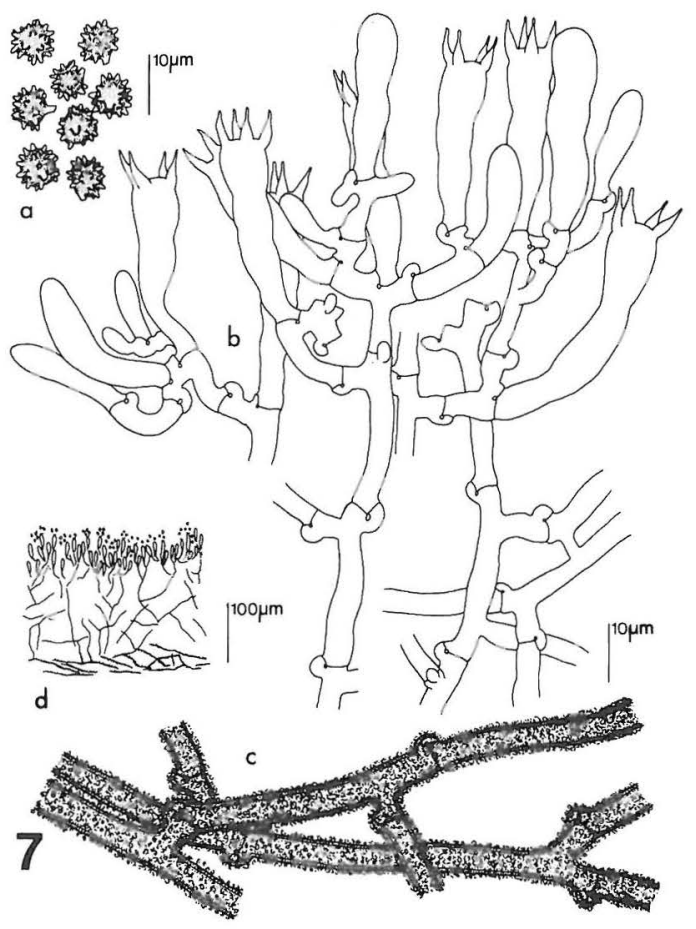

Fig. 7. Tomentella neobourdotii (I. Melo 7165, LISU): a. basidiospores, b. basidia and subhymenial hyphae, c. subicular hyphae, d. section through basidioma. 
(7.5YR 3/2) to dark reddish brown (5YR 3/2). Subiculum arachnoid, concolorous; without cordons. Margin indeterminate. Hyphal system monomitic; subicular hyphae with clamps, 5-7 $\mu \mathrm{m}$ diam., thick-walled, strongly encrusted, brown; subhymenial hyphae with clamps, 3-4 $\mu \mathrm{m}$ diam., thin-walled, sometimes minutely encrusted, pale brown. Basidia clavate, sinuous, $27-45 \times 5-8 \mu \mathrm{m}, 4$ up to $7 \mu \mathrm{m}$ long sterigmata. Basidiospores irregularly globose, 6-7 $\mu \mathrm{m}$, echinulate, pale brown.

Remarks: This species can be distinguished by its strongly incrusted subicular hyphae and the size and shape of its spores. So far known only from Spain, Alicante (Malençon \& Llimona 1983), Asturias (Dueñas \& Tellería 1988), Barcelona and Orense (Tellería 1984), Menorca (Tellería et al. 1997) and Tarragona (Llimona et al. 1998), its distribution is now extended to Portugal.

Material studied: Portugal. Beira Alta, Viseu, Parque Florestal de Fontelo, 29TNF9301, on Quercus robur L., 7.XI.1996 IM 7165 (LISU). Spain. Alava, Maestu, entre Maestu y Corres, El Ayedo, 30TWN4629, 850 m, on Quercus rotundifolia Lam., 6.XII.1987 IS 4242 (BIOFungi 2102); Zuya, Marquina, 30TWN1558, $680 \mathrm{~m}$, on Ulex sp., 5.XII.1987 IS 4207 (BIO-Fungi 876); Zuya, Zarate, 30TWN1657, $700 \mathrm{~m}$, on Quercus pyrenaica Willd., 19.IX.1992 IS 5711 (BIO-Fungi 7356). Asturias, Reserva Biológica de Muniellos, Tablizas, 29TPH8868, on Quercus petraea (Matt.) Liebl., 18.X.1983 MD 1242 (MA-Fungi 15467). León, Carretera Nacional VI, entre Villafranca del Bierzo y Pereje, km 415, 29TQH7822, $630 \mathrm{~m}$, on Castanea sativa Mill., 12.XII.1984 MD 3281 (MA-Fungi 15465); Posada de Valdeón, del Collado de Panderruedas a Posada de Valdeón, 30TUN4076, 1220 m, on Fagus sylvatica L., 20.X.1992 IM 5769 (LISU). Jaén, Parque Natural de Cazorla, Segura y las Villas, La Iruela, camino de Fresnedilla a la piscifactoría, 30SWH1303, 960 m, on Arbutus unedo L., 13.V.1990 MD 5622 (MA-Fungi 27488); ibidem, on Pinus pinaster Aiton, MD 5627 (MA-Fungi 27492); ibidem, on Quercus rotundifolia Lam., MT 10533 (MA-Fungi 37421). Lugo, Cervantes, Castillo de Frades, río de Cereixedo, 29TPH6740, 740 m, on Quercus pyrenaica Willd., 5.X.1994 IM 6322 (LISU). Madrid, Miraflores de la Sierra, 30TVL3518, on Quercus pyrenaica Willd., 23.XI.1979 (MA-Fungi 1147). Orense, Untes, 29TNG8688, on Quercus suber L., 26.XI.1982 MT 2135 (MA-Fungi 6716). Vizcaya, bajada de Urkiola a Ochandiano, 30TWN2870, on Pseudotsuga sp., 9.X.1983 IS 430 (BIO-Fungi 8048).

Tomentella ramosissima (Berk. \& M.A. Curtis) Wakef., Mycologia 52: 927. 1962. - Fig. 8 $\equiv$ Zygodesmus ramosissimus Berk. \& M.A. Curtis in Berk., Grevillea 3: 145. 1875

Basidioma resupinate, adnate to separable from the substrate, continuous, mucedinoid. Hymen-

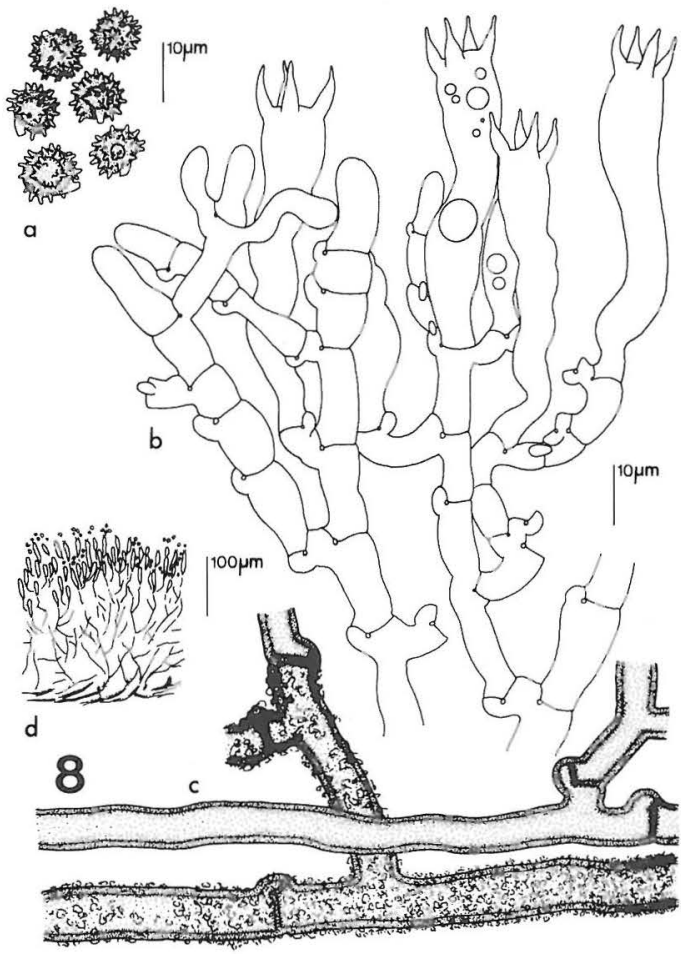

Fig. 8. Tomentella ramosissima (I. Melo 5040, LISU): a. basidiospores, b. basidia and subhymenial hyphae, c. subicular hyphae, d. section through basidioma.

ophore granulose, reddish brown (5YR 4/3) to dark reddish gray (5YR 4/2). Subiculum arachnoid, concolorous to darker than the hymenophore; without cordons. Margin indeterminate. Hyphal system monomitic; subicular hyphae with clamps, 6-10 $\mu \mathrm{m}$ diam., very thick-walled, sometimes minutely encrusted, dark brown to golden brown; subhymenial hyphae with clamps, 4-6 $\mu \mathrm{m}$ diam., thin to slightly thickwalled, often minutely encrusted, pale golden brown. Subhymenial and hymenial hyphae often with bluish green diffusates in $3 \% \mathrm{KOH}$. Basidia clavate, $35-60 \times 9-12 \mu \mathrm{m}, 4$ up to $7 \mu \mathrm{m}$ long sterigmata. Basidiospores globose to subglobose, 7-9 $\mu \mathrm{m}$ diam., echinulate to aculeate, golden brown.

Remarks: This species can be characterized by its wide, dark to golden brown subicular hyphae and the presence of a strong bluish green diffusate in $\mathrm{KOH}$ in the hymenial and subhymenial hyphae. Species relatively common, widely distributed in Spain: Asturias (Dueñas \& Tellería 
1988), Barcelona (Tellería 1984), Huesca (Melo et al. 1993), Menorca (Tellería et al. 1997), Segovia (Calonge 1968) and Tarragona (Llimona et al. 1998). There is only one reference from Portugal (Larsen 1974).

Material studied: Spain. Alava, Beotegui, 30TVN9371, $380 \mathrm{~m}$, on Clematis sp., 5.XI.1988 IS 4586 (BIO-Fungi 2469); Oyón, Labraza, bosque de Dueñas, 30TWN4913, $670 \mathrm{~m}$, on Pinus halepensis Mill., 7.XII.1987 IS 4446 (BIO-Fungi 2112); Valdegovia, Guinea, 30TVN9844, $800 \mathrm{~m}$, on Pinus sylvestris L., 17.V.1987 IS 3593 (BIO-Fungi 598); ibidem, on Pinus sylvestris L., 13.XI.1986 IS 2514 (BIO-Fungi 597). Asturias, Reserva Biológica de Muniellos, Tablizas, 29TPH8868, $700 \mathrm{~m}$, on Quercus petraea (Matt.) Liebl., 18.X.1983 MD 1169 (MA-Fungi 14661); ibidem, on unidentified wood, 19.X.1983 MD 1408 (MA-Fungi 14660). Avila, El Tiemblo, pinal de El Tiemblo, 30TUK7375, on Pinus sp., 27.X.1982 MT 1374 (MAFungi 7420); idem, MT 1389 (MA-Fungi 7419). Barcelona, Cabrils, 30TCH32, 31.X.1979 MT 148/79 (MAFungi 5688); ibidem, on Quercus suber L., 31.X.1979, MT (MA-Fungi 5675); idem, MT 159/79 (MA-Fungi 5679); idem, MT 161/79 (MA-Fungi 5681). Burgos, Vil-

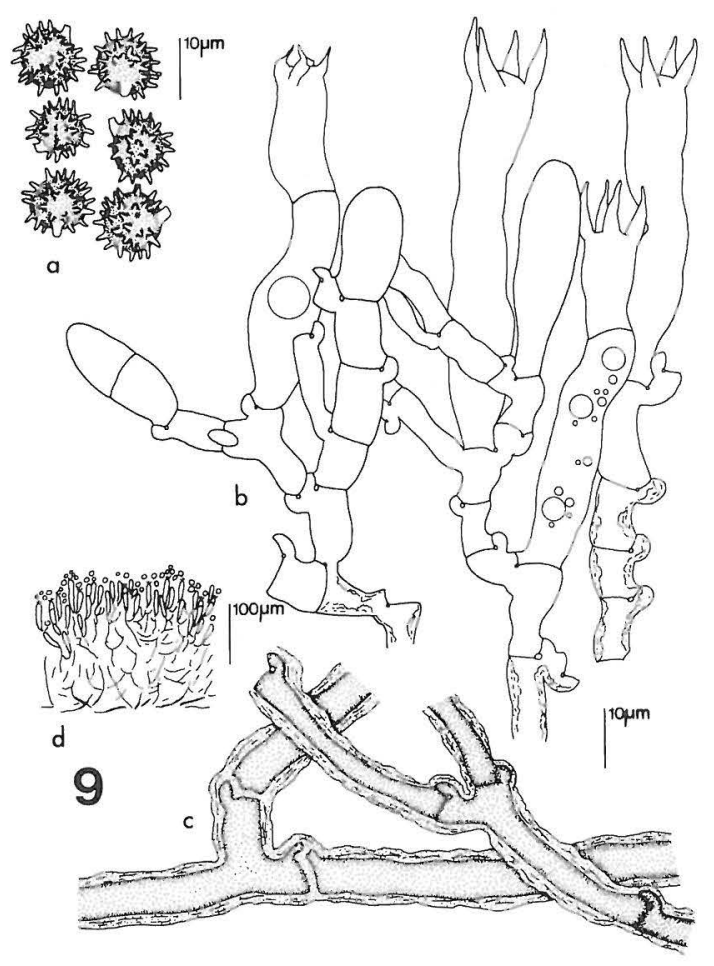

Fig. 9. Tomentella stuposa (I Salcedo 6880, BIO-Fungi 5866): a. basidiospores, b. basidia and subhymenial hyphae, c. subicular hyphae, d. section through basidioma. Note the strong swelling of the cell walls of the subhymenial and subicular hyphae in $3 \% \mathrm{KOH}$. lanueva de los Montes, 30TVN7033, $600 \mathrm{~m}$, on Pinus pinaster Aiton, 28.XI.1992 IS 6512 (BIO-Fungi 5202). Cantabria, Los Tojos, de Barcena Mayor al cruce de Los Tojos, junto al río Argoza Labor, 30TVN0179, $570 \mathrm{~m}$, 9.X.1986 MT 7761 (MA-Fungi 19025). Gerona, Pals, Pinedes dels Masos de Pals, 31TEG1548, 40 m, on Rosmarinus officinalis L., 9.XI.1995 IM 6753 (LISU). Huesca, Selva de Oza, 30TXN8941, 1160 m, on Abies alba Mill., 10.XI.1977 MT 758/77 (MA-Fungi 3703); Valle de Vio, Nerín, barranco de Flageta, 31TBH5617, on Pinus sylvestris L., 18.X.1989 MD 5345 (MA-Fungi 26462). Lérida, Puerto del Pontón, 30TUN3184, on Fagus sylvatica L., 8.XII.1984 MD 3177 (MA-Fungi 14630). Menorca, Mahón, S' Albufera, 31SFE0823, on Rosmarinus officinalis L., 15.XI.1990 IM 5115 (LISU); Mercadal, Binifamis, 31SFE0128, $60 \mathrm{~m}$, on Pinus halepensis Mill., 15.XI.1990 IM 5040 (LISU); ibidem, on grasses, 15.XI.1990 IM 5074 (LISU). Palencia, de Cervera de Pisuerga a Santibañez de Resoba, alrededores de Cervera, 30TUN7747, on Quercus pyrenaica Willd., 20.XI.1984 MD 2272 (MA-Fungi 15466).

Tomentella stuposa (Link) Stalpers, Stud. Mycol. 24: 86. 1984. - Fig. 9

$\equiv$ Sporotrichum stuposum Link, Mag. Ges. Naturf. Freunde, Berlin 3: 12. 1809).

= Tomentella ruttneri Litsch., Bull. Soc. Mycol. France 49: 67. 1933.

Basidioma resupinate, adherent, continuous, mucedinoid. Hymenophore smooth, reddish brown (5YR 5/3, 4/3). Subiculum dense, concolorous; without cordons. Margin indeterminate. Hyphal system monomitic; subicular hyphae with clamps, 6-8 $\mu \mathrm{m}$ diam., thick-walled, swelling quickly in $3 \% \mathrm{KOH}$, golden brown; subhymenial hyphae with clamps, 4-6 $\mu \mathrm{m}$ diam., densely ramified, with short articles, thin-walled, pale brown, swelling quickly too. Basidia cylindrical to clavate, sinuous, with transverse septa, $50-60 \times 10-12 \mu \mathrm{m}, 4$ up to $10 \mu \mathrm{m}$ long sterigmata. Basidiospores globose to subglobose, $8-11 \times 7-$ $10 \mu \mathrm{m}$, aculeate, pale brown.

Remarks: Similar to $T$. brevispina and $T$. bresadola. From the latter, T. stuposa can be recognized by the golden brown subicular hyphae together with the strong swelling of the cells walls in $\mathrm{KOH}$. This last characteristic and the larger spores distinguish T. stuposa from $T$. brevispina. So far only known from one locality in peninsular Spain, Lerida (Tellería \& Dueñas 1986, as T. ruttneri Litsch.).

Material studied: Spain. Alava, Cigoitia, Apodaca, 30TWN2152, $550 \mathrm{~m}$, on Phellinus sp., 2.X.1993 IS 6880 (BIO-Fungi 5866). Lérida, carretera de Sorbe al Puerto de la Bonaigua, 31TCH3326, $1550 \mathrm{~m}$, on Abies alba Mill., 4.VI.1984 MT 6104 (MA-Fungi 14485). 
Acknowledgements: We are indebted to Dr. Michael J. Larsen for improvements of the paper and for revising the manuscript.

\section{References}

Bertault, R. 1982: Contribution à la Flore Mycologique de la Catalogne. - Acta Bot. Barcinonen. 34: 1-35.

Blanco, M.N. 1991: Estudio taxonómico, corológico y ecológico de los Aphyllophorales s.l. (Basidiomycotina) del Parque Natural de Monfragüe (Extremadura). - Societat Catalana de Micología. Edi. especials 4:1186.

Brummitt, R.K. \& Powell, C.E. (eds.) 1992: Authors of Plant Names. - Royal Botanic Gardens, Kew. 732 pp.

Calonge, F.D. 1968: Estudios sobre hongos. 1. Algunos ejemplares colectados en Madrid y sus alrededores. Anales Inst. Bot. Cavanilles 26: 15-35 (1970).

Dueñas, M. 1991: Contribución al estudio de los Aphyllophorales de Ciudad Real y Toledo. - Bol. Soc. Micol. Madrid 15: 165-176.

Dueñas, M. \& Tellería, M.T. 1988: Catálogo de los Corticiáceos y Poliporáceos, s.l. (Aphyllophorales, Basidiomycotina (1990) de la micoflora cántabro-astur. - Ruizia 5: 1-164

Hjortstam, K., Tellería, M.T., Ryvarden, L. \& Calonge, F.D. 1981: Notes on the Aphyllophorales of Spain. II. - Nova Hedwigia 34: 525-538.

Kõljalg, U. 1996: Tomentella (Basidiomycota) and related genera in the Temperate Eurasia. - Synopsis Fungorum 9: 1-213.

Kõljalg, U. 1999: Molecular phylogeny of Thelephorales. 25 years of Mycology in Tübingen. - Annual Meeting of the "Gesellschaft für Mykologie und Lichenologie e. V. (CML)" and the "Sektion Mykologie unf Lichenologie der Deutschen Botanischen Gessellschaft (SML)" $3^{\text {rd }}-6^{\text {th }}$ June, 1999: 16.

Larsen, M.J. 1974: A Contribution to the Taxonomy of the Genus Tomentella. - Mycol. Mem. 4: 1-145.

Larsen, M.J. 1981: The Genus Tomentellastrum (Aphyllophorales, Thelephoraceae s. str.). - Nova Hedwigia 35: 1-16.
Llimona, X., Blanco, M.N., Dueñas, M., Gorris, M., Gràcia, E., Hoyo, P., Llistosella, J., Martí, J., Martín, M.P., Muntañola-Cvetkovic, M., Quadrada, R., Rocabruna, A., Salcedo, I., Sierra, D., Tabarés, M. \& Vila, J. 1998: Els fongs de Catalunya occidental segons les prospeccions recents. I. - Acta Bot. Barcinon. 45 (Homenatge a Oriol de Bolòs): 57-89.

Malençon, G. \& Bertault, R. 1971: Champignons de la Péninsule Ibérique. I. Explorations entre le Midi Valencien et le Montseny. - Acta Phytotax. Barcinon. 8: 5-68.

Malençon, G. \& Llimona, X. 1983: Champignons de la Péninsule Ibérique. VII. Flore vernale du SE: Basidiomycétes. - Anales Univ. Murcia Ci. 41: 3-89.

Melo, I., Salcedo, I. \& Tellería, M.T. 1998: Contribution to the knowledge of Tomentelloid Fungi in the Iberian Peninsula. - Folia Cryptog. Estonica. 33: 77 84.

Melo, I., Tellería, M.T. \& Dueñas, M. 1993: Catálogo de Aphyllophorales (Basidiomycotina) del Parque Nacional de Ordesa y Monte Perdido (Huesca). - Bol. Soc. Micol. Madrid 18: 19-40.

Munsell Soil Color Charts 1990: Macbeth Div. of Kollmogen Inst. Corp. Baltimore.

Tellería, M.T. 1980: Contribución al estudio de los Aphyllophorales españoles. - Biblioth. Mycol. 74.

Tellería, M.T. 1984: De Aphyllophoralibus in Hispania provenientibus ordinati commentarii I. - Bol. Soc. Micol. Castellana 8: 61-78.

Tellería, M.T. 1985: De Aphyllophoralibus in Hispania provenientibus ordinati commentarii III. - Anales Jard. Bot. Madrid 42: 49-60.

Tellería, M.T. \& Dueñas, M. 1986: De Aphyllophoralibus in Hispania provenientibus ordinati commentarii IV. - Anales Jard. Bot. Madrid 43: 3-7.

Tellería, M.T., Melo, I. \& Dueñas, M. 1997: An Annotated List of the Aphyllophorales of the Balearic Islands. - Mycotaxon 65: 353-377.

Torrend, C. 1913: Les Basidiomycètes des environs de Lisbonne et de la région de $\mathrm{S}$. Fiel (Beira Baixa). Brotéria, Sér. Bot. 11: 73-98. 